\title{
CRYSTALLOGRAPHIC PROPERTIES OF BULK GaN CRYSTALS GROWN AT HIGH PRESSURE ${ }^{\S}$
}

\author{
M. Leszczyński, I. Grzegory, M. BoćKowski, J. Jun, S. Porowski \\ High Pressure Research Center, Polish Academy of Sciences \\ Sokołowska 29/37, 01-142 Warszawa, Poland \\ J. JASIŃSKI AND J.M. BARANOWSKI \\ Institute of Experimental Physics, Warsaw University \\ Hoża 69, 00-681 Warszawa, Poland
}

\begin{abstract}
Gallium nitride bulk crystals grown at about $15 \mathrm{kbar}$ and $1500 \mathrm{~K}$ have been examined by using the high resolution X-ray diffractometry. An analysis of a set of the rocking curves of various Bragg reflections enabled us to estimate a dislocation density. For the crystals of dimensions lower than about $1 \mathrm{~mm}$ it is lower than $10^{-5} \mathrm{~cm}^{-2}$. For bigger samples the crystallographic quality worsens. With an application of the reciprocal lattice mapping we could distinguish between internal strains and mosaicity which are both present in these crystals.' The results for the bulk crystals are compared with those for epitaxial layers.
\end{abstract}

PACS numbers: $61.50 . \mathrm{Cj}, 61.72 . \mathrm{Dd}$

\section{Introduction}

Gallium nitride is the most promising material for the optoelectronics operating in blue and ultraviolet spectrum. Its recent popularity is gained due to the $3.4 \mathrm{eV}$ direct band-gap, low dislocation mobility and lack of success with the other candidates. Also a commercial success of the $\mathrm{GaN}$-based blue-light emitting diode has stimulated the research. Majority of the works are performed on epitaxial layers grown by molecular beam epitaxy or metalorganic chemical vapour deposition. Unfortunately, no GaN-lattice-matched substrates are available and the layers possess a very high density of misfit dislocations. Such materials can hardly serve for a construction of a blue laser which is a main goal of the research. Growth of the bulk crystals, which could be used as substrates for homoepitaxy, is difficult because of decomposition of $\mathrm{GaN}$ at about $1000^{\circ} \mathrm{C}$ at normal pressure. Therefore, we have examined the growth of GaN bulk crystals at pressures of 1-15 kbar for more than 10 years [1-4]. At present, our samples may have a dimension up to a few millimetres. This work is devoted to the crystallographic properties of these

§ This work is supported by the grant of the State Committee for Scientific Research (Republic of Poland) no. 288/P4/94/06. 
crystals examined by using the high resolution $\mathrm{X}$-ray diffraction. We show the results for 3 typical bulk crystals (hexagonal plates) of different size $(0.5,2,4 \mathrm{~mm}$ ) compared with the $2 \mu \mathrm{m}$ layers grown by metalorganic chemical vapour deposition (MOCVD) on sapphire (16\% lattice mismatch) and on $\mathrm{SiC}(3.4 \%$ mismatch) substrates.

\section{Dislocation density}

The full width at half maximum (FWHM) of the diffraction peak (characterized by Miller indices $h k l$ ) can be approximated by the following equation [5]:

$$
\beta_{\mathrm{m}}^{2}(h k l)=\beta_{0}^{2}(h k l)+\beta_{a}^{2}(h k l)+\beta_{\varepsilon}^{2}(h k l),
$$

where $\beta_{\mathrm{m}}(h k l)$ is the FWHM of the measured rocking curve, $\beta_{0}(h k l)$ is the intrinsic rocking curve (as we used a primary $\mathrm{X}$-ray beam of 5 arcsec divergence we could neglect an instrument function), $\beta_{\mathrm{a}}(h k l)$ is the broadening caused by angular rotation at dislocations and

$$
\beta_{\mathrm{a}}(h k l) \approx 6 \ln \left(2 b^{2} D\right),
$$

$D$ is the dislocation density, $b$ is the length of the Burgers vector, $\beta_{\varepsilon}(h k l)$ is the broadening caused by the strain surrounding dislocations

$$
\beta_{\varepsilon}(h k l) \approx 0.1 b^{2} D\left|\ln \left(2 \times 10^{-7} \mathrm{~cm} \sqrt{D}\right)\right| \tan ^{2} \Theta,
$$

$\Theta$ is the Bragg angle for $h k l$ reflection.

The above equations for $\beta_{\mathrm{a}}$ and $\beta_{\varepsilon}$ depend on the character of the dislocations, but not very strongly and they can well serve for a determination of a range

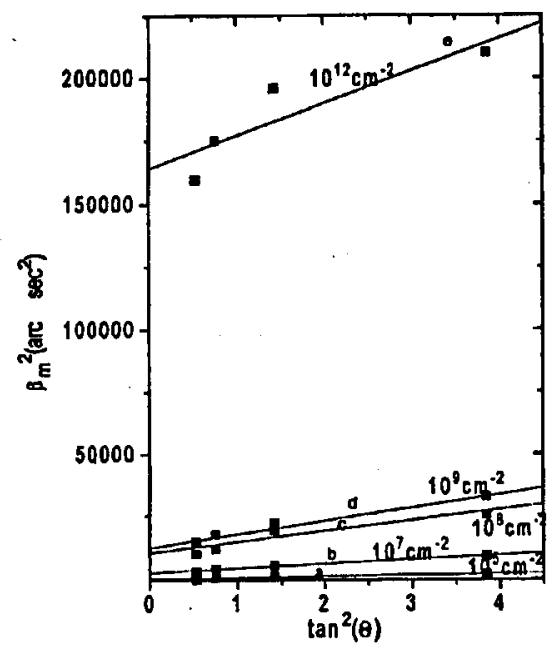

Fig. 1. The square of broadening of rocking curves from GaN samples for different Bragg angles. $a-0.5 \mathrm{~mm}$ bulk, $b-2 \mathrm{~mm}$ bulk, $c-4 \mathrm{~mm}$ bulk, $d-$ layer on SiC, $e$ - layer on sapphire. The values inside the figure indicate the upper limit of dislocation density for a corresponding sample. 
of magnitude of $D$. Figure 1 shows the dependence of the square of rocking-curve broadening versus $\tan ^{2} \Theta$ (different Bragg reflections). These results served for an evaluation of dislocation density basing on Eq. (1). The values of $D$ presented in Fig. 1 should be treated as their upper limits because we do not take into account other defects or inhomogeneities which may broaden the rocking curves. Additionally, for the smallest sample it was not possible to a void the penetration of X-rays through the crystal edges, especially for asymmetric reflections. In such a case an $\mathrm{X}$-ray diffraction becomes very complicated and it is practically impossible to evaluate the peak broadening due to that factor. For the layer grown on $\mathrm{SiC}$ a density of misfit dislocations is of about $10^{11}-10^{12} \mathrm{~cm}^{-2}$ and of about $10^{13}-10^{14} \mathrm{~cm}^{-2}$ for the layer grown on sapphire (both layers are relaxed). The values of $D$ given in Fig. 2 for both layers correspond to the density of threading dislocations.

\section{Reciprocal lattice mapping}

The reciprocal lattice maps (intensity iso-contours) are created by performing independent scans of $\omega$ (sample rocking) and $2 \Theta$ (reflected-intensity-counter rotation) [6]. Figure 2 shows such maps. For the smallest bulk sample the result is very close to the one of the perfect crystal. For $2 \mathrm{~mm}$ sample we may observe a

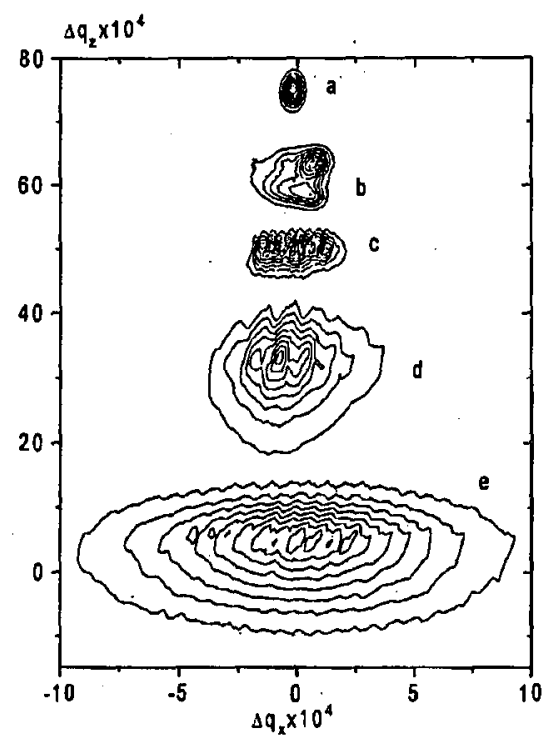

Fig. 2. Reciprocal space maps of (00.4) $\mathrm{Cu} K_{\alpha_{1}}$ reflection from GaN samples. $a-$ $0.5 \mathrm{~mm}$ bulk, $b-2 \mathrm{~mm}$ bulk, $c-4 \mathrm{~mm}$ bulk, $d-$ layer on SiC, $e$ - layer on sapphire. $\Delta q_{z}$ is a change of length of the reciprocal lattice vector in the direction perpendicular to the surface (representing strain in this direction), $\Delta q_{x}-$ in the parallel direction (mosaicity). Both axes are marked in units of $\lambda / 2 d$ ( $\lambda-\mathrm{X}$-ray wavelength, $d-$ lattice spacing). 
strain (elongation along $\Delta q_{z}$ axis) of about $10^{-4}$. The $4 \mathrm{~mm}$ crystal is mosaic (a series of peaks along $\Delta q_{x}$ axis). It can be also seen that the layer on $\mathrm{SiC}$ is slightly more strained than the $4 \mathrm{~mm}$ bulk sample but has a similar degree of mosaicity. The layer on sapphire is highly mosaic.

\section{Summary}

The high resolution X-ray diffraction applied for an investigation of GaN bulk crystals and epitaxial layers enabled us to evaluate the dislocation density, strains and mosaicity. We observed a creation of strains and mosaic structure for millimetre size bulk crystals. A deteriorating influence of the lattice mismatch is presented by a comparison of samples with $3.4 \%$ and $16 \%$ mismatch.

\section{References}

[1] J. Karpinski, J. Jun, S. Porowski, J. Cryst. Growth 66, 1 (1984).

[2] S. Porowski, I. Grzegory, J. Jun, in: High Pressure Chemical Synthesis, Eds. J. Jurczak, B. Baranowski, Elsevier, Amsterdam 1989, p. 21.

[3] I. Grzegory, S. Krukowski, Phys. Scr. Vol. T 39, 249 (1991).

[4] M. Leszczynski, I. Grzegory, M. Bockowski, J. Cryst. Growth 126, 601 (1993).

[5] J.E. Ayers, J. Cryst. Growth 135, 71 (1994).

[6] P. Fewster, Philips J. Res. 47, R1275 (1993). 